\title{
PERIODIC ORBITS AS A PROBE TO REVEAL EXOTIC STATES IN VIBRATIONALLY EXCITED MOLECULES: THE SADDLE-NODE STATES
}

\author{
STAVROS C. FARANTOS \\ Institute of Electronic Structure and Laser, FORTH, and Department of \\ Chemistry, University of Crete, Iraklion, Crete 711 10, Greece.
}

(Received 8 July, 1992)

\begin{abstract}
We present theoretical results which show the existence of isomerizing localized quantum states above potential barriers for the excited ${ }^{1} \mathrm{~B}_{2}$ states of $\mathrm{SO}_{2}$ and $\mathrm{O}_{3}$. These states are assigned by periodic orbits which emerge from saddle-node bifurcations, the characteristic of which is the simultaneous generation of two families of periodic orbits one stable and one unstable. Similar isomerizing states and bifurcations have been found for other molecules, and this leads to the conclusion, that the appearance of saddle-node type states may be a generic phenomenon for molecular potentials with barriers.
\end{abstract}

KEY WORDS: Vibrational spectroscopy, quantum dynamics, quantum chaos and localization, periodic orbits, saddle-node bifurcations.

\section{INTRODUCTION}

The spectroscopy of vibrationally highly excited molecules has been the subject of intense research in the last years. The methods of stimulated emission pumping (SEP), ${ }^{1}$ vibrational predissociation spectroscopy (VPS), and supersonic jet FTIR and laser spectroscopy ${ }^{2}$ are among those which have improved significantly the resolution of the spectra. The theoretical calculations have also been advanced by using the time dependent formulation of photoabsorption and Raman spectroscopy, ${ }^{3}$ the discrete variable representation method (DVR), ${ }^{4}$ as well as other grid methods for solving the time dependent Schrödinger equation. ${ }^{5}$

Theoretical and experimental spectroscopy are aiming to extract the dynamics of the energized molecules, results which shed light to the very interesting topics of the energy redistribution and quantum chaos. ${ }^{6}$ If we take in account that regular/chaotic states are usually localized/diffused in configuration space, we can infer the practical importance of understanding the dynamics of vibrationally excited molecules for the state specific chemistry.

Acetylene, ${ }^{7} \mathrm{NO}_{2},{ }^{8} \mathrm{Na}_{3},{ }^{9,10} \mathrm{HCN},{ }^{11-13} \mathrm{O}_{3},{ }^{14-16} \mathrm{CS}_{2},{ }^{17}$ and $\mathrm{SO}_{2}{ }^{18}$ are molecules which have been studied in association to the problem of quantum chaos. One important outcome of these studies is that, in spite of the strong classical chaotic behaviour, the quantum dynamics of the system can show an appreciable ordering due to the 
localization of the eigenstates along periodic orbits. ${ }^{19}$ This results in observing regular progressions in low resolution spectra.

The correspondence among quantum states and classical mechanical objects, such as tori or reduced dimension tori ${ }^{10}$ periodic orbits, ${ }^{20-22}$ and the unstable and stable manifolds of unstable periodic orbits, ${ }^{23}$ has been found in numerous calculations. Particularly, periodic orbits have been studied by mathematicians and astronomers for almost a century. Poincare was the first to emphasize that the complex dynamics of non linear systems can be explored by periodic orbits. ${ }^{24}$ Now, mathematical theorems and numerical techniques allow a systematic search for locating periodic orbits and following their evolution with the total energy. 25,26

In the last years, we have carried out a systematic search of periodic orbits for molecular potentials by constructing bifurcation diagrams, ${ }^{12,16,27-29}$ i.e. plots of the initial conditions of periodic orbits with the total energy. This allows us to visualize the structure of phase space, i.e. to locate the regions with the less unstable motion. We assume that the quantum states are localized in such regions of phase space, and extract them by solving the time dependent Schrödinger equation with an initial wavepacket centered on a particular periodic orbit.

The principal families of periodic orbits are those which emerge from the extrema of the potential energy surface. Then, as the energy of the system increases more periodic orbits appear by bifurcations of the old ones. However, new families of periodic orbits may appear suddenly at some energy and they always come in pairs, one stable and one unstable family, irrespectively to how unstable the phase space has been at lower energies. They have been given several names such as periodic orbits "out of nowhere",$^{30}$ irregular, ${ }^{31}$ rotational, ${ }^{12,29}$ tangent, or saddle-node ${ }^{25}$ In our studies for the molecules of $\mathrm{HCN},{ }^{12}$ and $\mathrm{ArCO},{ }^{29}$ we have found saddle-node periodic orbits to appear mainly above isomerization barriers.

In this article we present new results for the excited ${ }^{1} \mathrm{~B}_{2}$ electronic state of $\mathrm{SO}_{2}$. It is shown that even a tiny potential barrier which separates two asymmetric minima is enough to generate saddle-node periodic orbits which correspond to bound eigenfunctions of similar morphology, and thus, we name them saddle-node states. We compare the above results with calculations on the ${ }^{1} \mathrm{~B}_{2}$ excited electronic state of ozone. In the present work, the recent calculation ${ }^{32}$ of the autocorrelation function of ozone are extended to longer times, and the first resonance states are extracted. This molecule is strongly chaotic but some structure in the autocorrelation function can be analyzed by saddle-node periodic orbits. These findings point out that the existence of saddle-node states is typical of molecules with potential barriers.

\section{THE PERIODIC ORBIT-TIME DEPENDENT SCHRÖDINGER EQUATION METHOD (PO-TDSE)}

We adopt a time dependent formalism as developed by Heller, ${ }^{3}$ since this allows a more immediate comparison between classical and quantum mechanics. We have recently reviewed the basic theory of periodic orbits and their bifurcations. ${ }^{33}$ Here we concentrate on saddle-node bifurcations. 
We can consider a saddle-node bifurcation as the coalescence of two periodic orbits at some particular value of the total energy. For 2-D systems it can be proved that the two periodic orbits are stable and unstable respectively, and at the bifurcation point all eigenvalues of the monodromy matrix are equal to one with degenerate eigenvectors.

To find the influence of a particular periodic orbit in quantum mechanics we solve the time dependent Schrödinger equation with an initial wavepacket, $\phi(\mathbf{Q}, 0)$, localized on the periodic orbit;

$$
i \hbar \frac{\partial \phi(\mathbf{Q}, \mathrm{t})}{\partial \mathrm{t}}=\hat{\mathrm{H}} \phi(\mathbf{Q}, \mathrm{t})
$$

$\hat{H}$ is the Hamiltonian of the system, and $\mathbf{Q}$ the coordinates. For short time the wavepacket follows the classical orbit, but for longer times the influence of the neighboring region will become apparent. In chaotic regions the wavepacket will be spread, quickly deviating from the periodic orbit.

The propagation of the wayepacket $\phi(t)$ in time, is obtained with several schemes: ${ }^{5}$

1. Second order difference method.

2. The split operator algorithm.

3. Representation of the propagator operator in Chebyshev series, and

4. the Lanczos method.

The second order difference method (SOD) is based on a Taylor expansion of the time propagator,

$$
\hat{\mathrm{U}}=\exp (-\mathrm{i} \Delta \mathrm{t} \hat{\mathrm{H}} / \hbar) \approx 1-\mathrm{i} \Delta \mathrm{t} \hat{\mathrm{H}} / \hbar-\frac{1}{2} \Delta \mathrm{t}^{2} \hat{\mathrm{H}}^{2} / \hbar^{2},
$$

and on symmetrizing appropriately in order to make the algorithm stable:

$$
\phi_{\mathrm{n}+1}(\mathbf{Q})-\phi_{\mathrm{n}-1}(\mathbf{Q})=\left(\hat{\mathrm{U}}-\hat{\mathrm{U}}^{+}\right) \phi_{\mathrm{n}}(\mathbf{Q}),
$$

where $\hat{\mathrm{U}}^{+}$is the complex conjugate of $\hat{\mathrm{U}}$. This procedure leads to the result,

$$
\phi_{\mathrm{n}+1}=\phi_{\mathrm{n}-1}-\frac{2 \mathrm{i}}{\hbar} \Delta \mathrm{tH} \phi_{\mathrm{n}} .
$$

$\Delta \mathrm{t}$ is the time elapsed between the $n$ and $n+1$ iterations.

The split operator procedure (SOP) proposed by Feit et al. ${ }^{34}$ is based on the equation,

$$
\phi\left(\mathbf{Q}, \mathrm{t}_{0}+\Delta \mathrm{t}\right)=\exp \left(\mathrm{i} \Delta \mathrm{t} \hbar \nabla^{2} / 4 \mu\right) \exp (-\mathrm{i} \Delta \mathrm{t} \mathrm{V} / \hbar) \exp \left(\mathrm{i} \Delta \mathrm{t} \hbar \nabla^{2} / 4 \mu\right) \phi\left(\mathbf{Q}, \mathrm{t}_{0}\right) .
$$

$V$ is the potential and $\mu$ the reduced mass. In this article, we employ the above two schemes, SOD and SOP, for the solution of the Schrödinger equation. 
From the evolution of the wavepacket the autocorrelation function,

$$
\mathrm{C}(\mathrm{t})=\langle\phi(\mathbf{Q}, \mathrm{t}=0) \phi(\mathbf{Q}, \mathrm{t})\rangle,
$$

is computed, the Fourier transform of which

$$
\mathrm{I}(\mathrm{E})=\frac{1}{2 \pi \hbar} \int_{-\infty}^{\infty} \exp (\mathrm{iEt} / \hbar) \mathrm{C}(\mathrm{t}) \mathrm{dt},
$$

gives the spectrum associated with the initial wavepacket.

The eigenfunctions, $\mathrm{I}>$, can be extracted by first estimating the eigenvalues, $E_{n}$, from the spectrum, Equation (7), and then by computing the integral;

$$
\text { I } \mathrm{n}>=\frac{1}{\mathrm{c}_{\mathrm{n}}} \int_{-\infty}^{\infty} \exp \left(\mathrm{iE}_{\mathrm{n}} \mathrm{t} / \hbar\right) \phi(\mathrm{t}) \mathrm{dt} \text {. }
$$

In the present work the calculations are performed in the symmetric and antisymmetric stretch space with the bending motion frozen. This is a good approximation for $\mathrm{SO}_{2}$ as Hoy and $\mathrm{Brand}^{35}$ have shown, but not for $\mathrm{O}_{3}\left({ }^{1} \mathrm{~B}_{2}\right)$. Recent 3-D quantum mechanical calculation $s^{36}$ have shown the importance of the bending mode. However, here we are interested in studying the role of the saddle-node periodic orbits in quantum mechanics, and thus, the problem is simplified by restricting the calculations in the 2-D space.

\section{RESULTS}

\subsection{Saddle-node vibrational eigenstates of $\mathrm{SO}_{2}\left({ }^{1} \mathrm{~B}_{2}\right)$}

For $\mathrm{SO}_{2}$ we use the Hamiltonian:

$$
\begin{gathered}
\mathrm{H}=\frac{1}{2} \omega_{1}\left(\mathrm{Q}_{1}^{2}+\mathrm{P}_{1}^{2}\right)+\frac{1}{2} \omega_{3}\left(\mathrm{Q}_{3}^{2}+\mathrm{P}_{3}^{2}\right)+\frac{1}{6} \mathrm{f}_{111} \mathrm{Q}_{1}^{3}+\frac{1}{2} \mathrm{f}_{133} \mathrm{Q}_{1} \mathrm{Q}_{3}^{2}+\frac{1}{24} \mathrm{f}_{1111} \mathrm{Q}_{1}^{4}+ \\
{ }_{4}^{\frac{1}{4}} \mathrm{f}_{1133} \mathrm{Q}_{1}^{2} \mathrm{Q}_{3}^{2}+\frac{1}{24} \mathrm{f}_{3333} \mathrm{Q}_{3}^{4}+\frac{\mathrm{B} \omega_{3} \exp (\rho)}{\exp (\rho)-\rho-1} \exp \left\{-[\exp (\rho)-\rho-1] \mathrm{Q}_{3}^{2} / 2 \mathrm{~B}\right\}+ \\
\mathrm{C} \exp \left[-\mathrm{D}_{1}\left(\mathrm{Q}_{1}-\mathrm{Q}_{10}\right)^{2}-\mathrm{D}_{3}\left(\mathrm{Q}_{3}-\mathrm{Q}_{30}\right)^{2}\right] .
\end{gathered}
$$

$\mathrm{Q}_{1}$ and $\mathrm{Q}_{3}$ are the symmetric and antisymmetric stretch normal coordinates respectively. The potential has been produced by Hoy and Brand ${ }^{35}$ and the parameters have been fitted with the data of the experimental spectra. We have added the last Gaussian function to eliminate a deep hole in the potential, which, however, does not change the potential in the fitted region of the minima and saddle. The potential function is shown in Figure 1. The saddle point is at $\left(\mathrm{Q}_{1}, \mathrm{Q}_{3}\right)=(0,0)$ and $0.0175 \mathrm{eV}$ above the minima.

We first search for saddle-node bifurcations at energies above the potential barrier. Because of the symmetry of the potential with respect to $Q_{3}$ the isomerizing periodic orbits, those which go over the barrier, satisfy the condition $\mathrm{P}_{1}=0$. This condition facilitates the location of the periodic orbits. Figure 2 is a bifurcation diagram showing three pairs of families of periodic orbits found for $\mathrm{SO}_{2}$. The dots denote the 


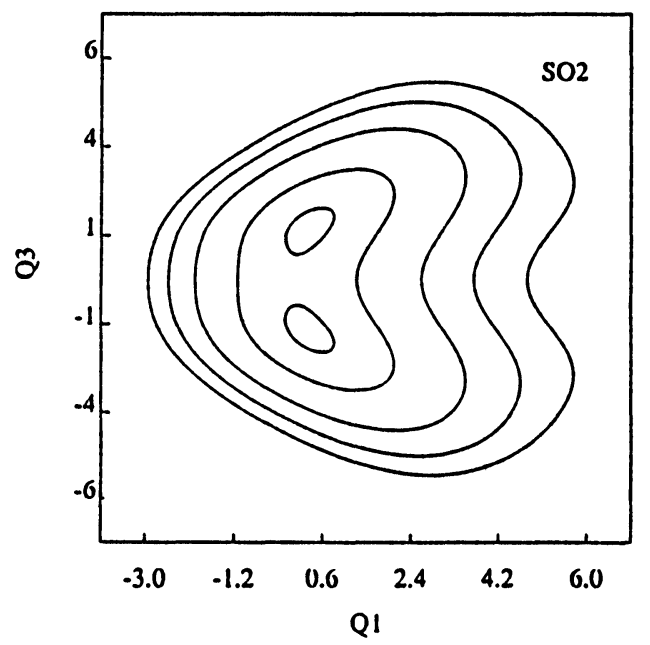

Figure 1 Potential energy surface of the ${ }^{1} \mathrm{~B}_{2}$ state of $\mathrm{SO}_{2}$. Contours are at $0.01,0.1,0.3,0.5$, and 0.7 eV. $Q_{1}$ and $Q_{3}$ are the symmetric and antisymmetric stretch normal coordinates.

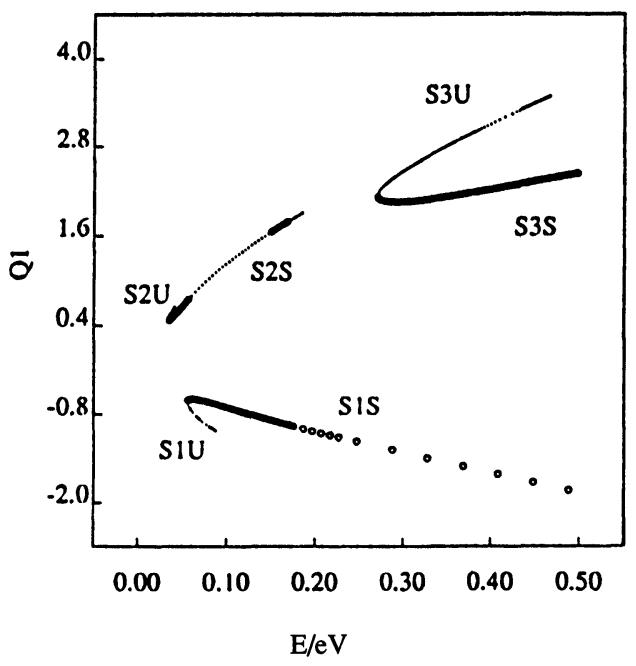

Figure 2 Bifurcation diagram showing three saddle-node bifurcations. Open circles denote stable periodic orbits and dots unstable periodic orbits.

unstable periodic orbits and the open circles the stable ones. Propagation of the unstable branches beyond the energies shown becomes difficult because of the quick increase of the instability. The located families are of the shortest periods and are expected to be important in quantum mechanics. Initial conditions for representative periodic orbits are given in Table 1.

The bifurcation diagram provides a sketch of the main characteristics of phase space, and points out possible quantum mechanical peculiarities. The eigenfunctions 
Table 1 Initial conditions of the saddle-node periodic orbits found for $\mathrm{SO}_{2}\left({ }^{1} \mathrm{~B}_{2}\right) . T$ is the period, $E$ the total energy, and the labels of periodic orbits are described in Figure 2.

\begin{tabular}{lllllll}
\hline$P O$ & $E / e V$ & $T / t . u$. & $Q_{I}$ & $Q_{3}$ & $P_{I}$ & $P_{3}$ \\
\hline S1S & 0.05558796 & 181.47 & -0.62317375 & 0.0 & 0.0 & 0.60642864 \\
S1U & 0.08586104 & 203.06 & -1.00766419 & 0.0 & 0.0 & 0.28266944 \\
S2S & 0.03938441 & 216.38 & 0.50477074 & 0.0 & 0.0 & 0.45739227 \\
S2U & 0.03557633 & 237.24 & 0.55027829 & 0.0 & 0.0 & 0.21319093 \\
S3S & 0.26978454 & 100.71 & 2.11681802 & 0.0 & 0.0 & 1.18248253 \\
S3U & 0.26956144 & 101.38 & 2.13777982 & 0.0 & 0.0 & 1.14237418 \\
\hline
\end{tabular}

are symmetric or antisymmetric with respect to $\mathrm{Q}_{3}$. Thus, in order to locate the antisymmetric ones we prepare an initial state as a difference of two Gaussian functions centered at the turning points of the periodic orbits. Similarly, to locate the symmetric eigenstates we take the sum of two equivalent Gaussians.

In Figure 3 we show the quantum spectrum produced by an initial wavepacket located on the periodic orbit S3S. We do the same for the periodic orbit of S1S type. The highest peaks are due to eigenfunctions which have the same topology as the periodic orbits. In Figure 4 we show two eigenfunctions with the periodic orbits superimposed. It is interesting to note that these two states which correspond to the energies of $0.31 \mathrm{eV}$ and $0.43 \mathrm{eV}$ respectively have the same number of nodes.

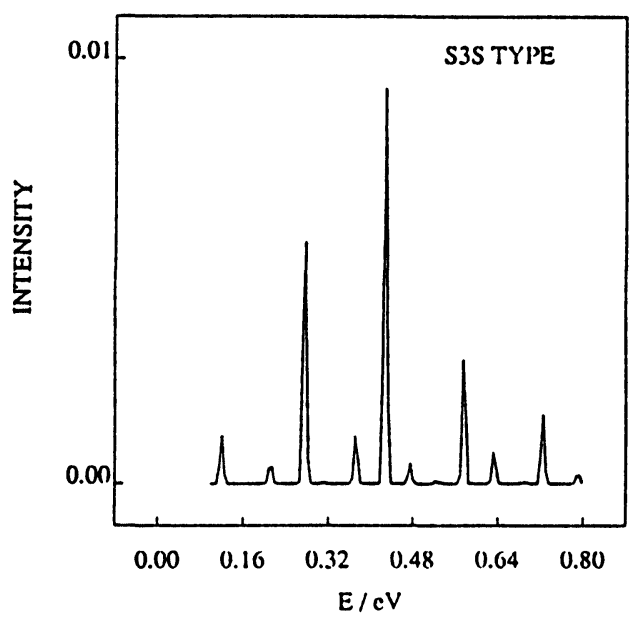

Figure 3 Quantum mechanical spectrum produced from an antisymmetric initial wavepacket made with two Gaussians centered on a periodic orbit of S3S type and with energy $0.43 \mathrm{eV}$.

We have examined other eigenfunctions which have one or more nodes in the $\mathrm{Q}_{1}$ coordinate but all of them keep the characteristic morphology of the S1 or S3 periodic orbits. It is also worth mentioning that for the stable branches the periods of periodic orbits decrease with the energy. This is characteristic of the rotating type states above 

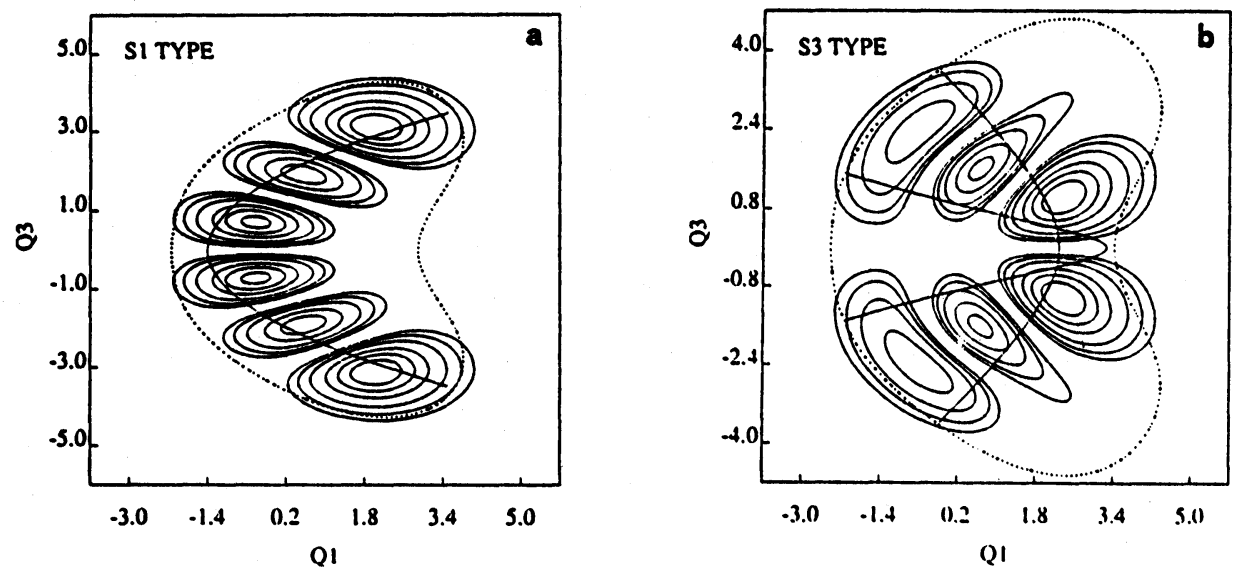

Figure 4 Two characteristic eigenfunctions assigned by the (a) S1S, and (b) S3S and S3U types periodic orbits. Eigenvalues are equal to (a) $0.31 \mathrm{eV}$ and (b) $0.43 \mathrm{eV}$.

barriers, and therefore they could be distinguished in an experimental spectrum. In fact, this is in agreement with the conclusions of Hoy and Brand, ${ }^{35}$ that is, the spectrum is explained by a double well potential in the antisymmetric coordinate which introduces a positive anharmonicity.

The morphology of the vibrational eigenfunctions of $\mathrm{SO}_{2}\left({ }^{1} \mathrm{~B}_{2}\right)$ reveals that the regions of phase space described by the periodic orbits $\mathrm{S} 1$ and $\mathrm{S} 3$ dominate in the behaviour of the molecule. This is further supported by plotting Poincare surfaces of sections. In Figure 5 we see that for this system the regularity increases with the energy. Moving from the energy of $0.21 \mathrm{eV}$ to $0.42 \mathrm{eV}$ the chaotic area shrinks
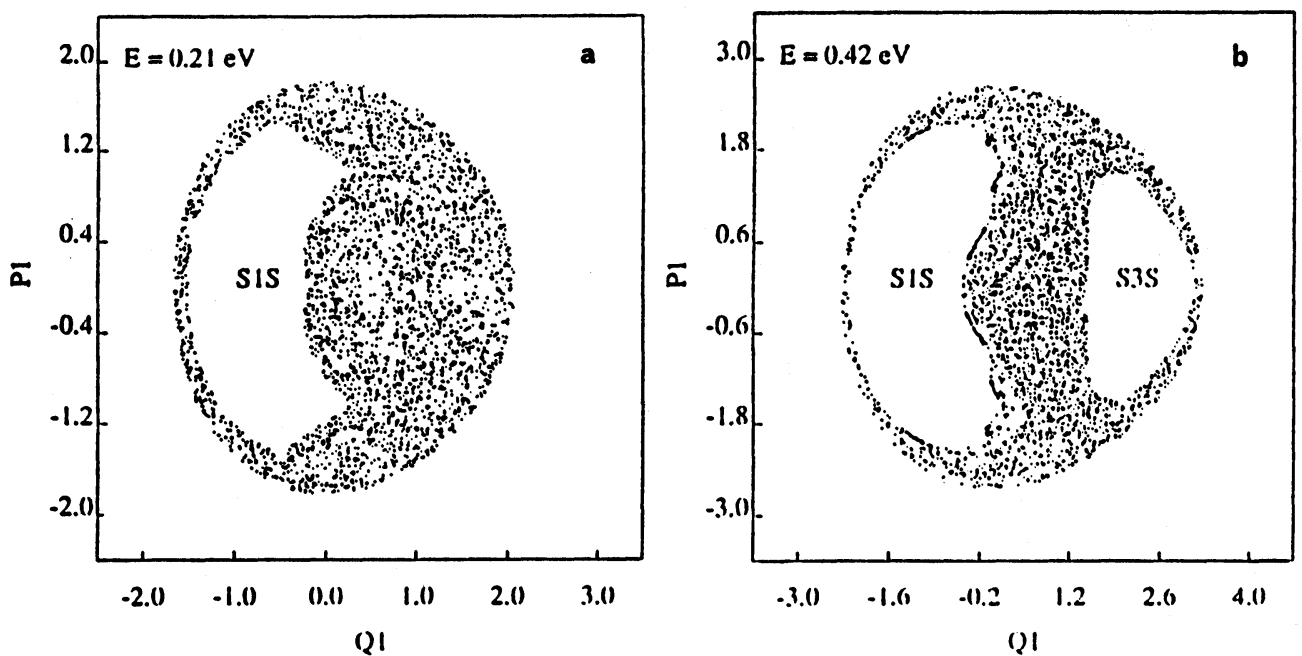

Figure 5 Poincare surfaces of sections produced by one chaotic trajectory at each energy. $\left(Q_{3}=0\right.$, $\left.\mathrm{P}_{3}>0\right)$. 
because of the S3S saddle-node periodic orbits. The stability region around them increases with the energy. This demonstrates why we believe that saddle-node bifurcations are important for molecules.

\subsection{Saddle-node resonances of $\mathrm{O}_{3}\left({ }^{1} \mathrm{~B}_{2}\right)$}

For ozone we use the Hamiltonian operator:

$$
\hat{\mathrm{H}}=-\frac{\hbar^{2}}{2 \mu} \frac{\partial^{2}}{\partial \mathrm{R}_{1}^{2}}-\frac{\hbar^{2}}{2 \mu} \frac{\partial^{2}}{\partial \mathrm{R}_{2}^{2}}-\frac{\hbar^{2} \cos \phi}{m_{o}} \frac{\partial^{2}}{\partial \mathrm{R}_{1} \partial \mathrm{R}_{2}}+\mathrm{V}\left(\mathrm{R}_{1}, \mathrm{R}_{2}\right),
$$

$\mathrm{R}_{1}$ and $\mathrm{R}_{2}$ are the two bond lengths of $\mathrm{O}-\mathrm{O}$, and the angle between them $\phi$, is kept fixed at $2.0245819 \mathrm{rad} . m_{\mathrm{o}}$ is the atomic mass of oxygen and $\mu$ the reduced mass of two oxygen atoms. The potential function is shown in Figure 6. The saddle point is $1.006 \mathrm{eV}$ above the minima, and it is located above the dissociation channels.

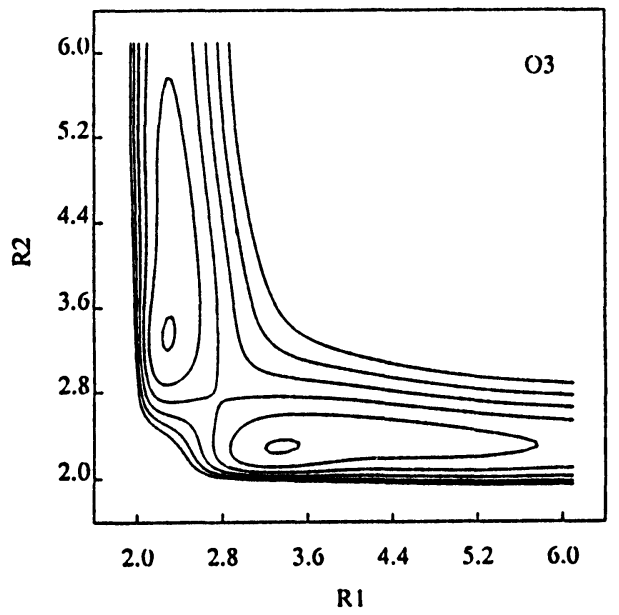

Figure 6 Potential energy surface of the ${ }^{1} \mathrm{~B}_{2}$ state of $\mathrm{O}_{3}$. Contours are at $0.5,1.0,1.5,2.0,2.5$, and $3.0 \mathrm{eV} . \mathrm{R}_{1}$ and $\mathrm{R}_{2}$ are the two $\mathrm{O}-\mathrm{O}$ bond lengths in Bohrs.

In reference [32] we presented the quantum autocorrelation function by integrating the Schrödinger equation up to $220 \mathrm{fs}$. These calculations have now been extended up to $440 \mathrm{fs}$. In Figure 7 the autocorrelation function is shown and the labels a,c,d,e, and $f$ of the peaks correspond to the periodic orbits found for this system (Figure 8). The assignment of the peaks is based on the agreement between the recurrence times of the wavepacket and the periods or half periods of the periodic orbits. Since, most periodic orbits are symmetric with respect to the ridge of the potential we expect the first recurrence to occur at half period. An exception is the periodic orbit $\mathrm{d}$, which returns to its initial coordinate values after a complete period. We have given the name of arrow and bow for this type of periodic orbits. The orbit e is as $\mathrm{d}$ but asymmetric, and it is obtained by integrating forward (solid line) and backward 


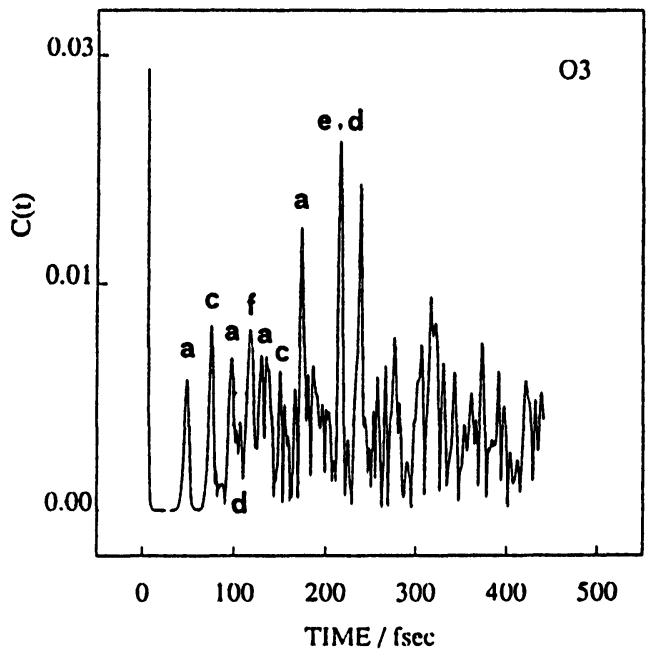

Figure 7 The quantum mechanical autocorrelation function produced with a Gaussian initial wavepacket, which describes the ground electronic vibrational state of ozone in the stretching coordinates. ${ }^{32}$ The labels, a,c,d,e,f, are the same with those of periodic orbits shown in Figure 8. These periodic orbits are associated with the resonance states.
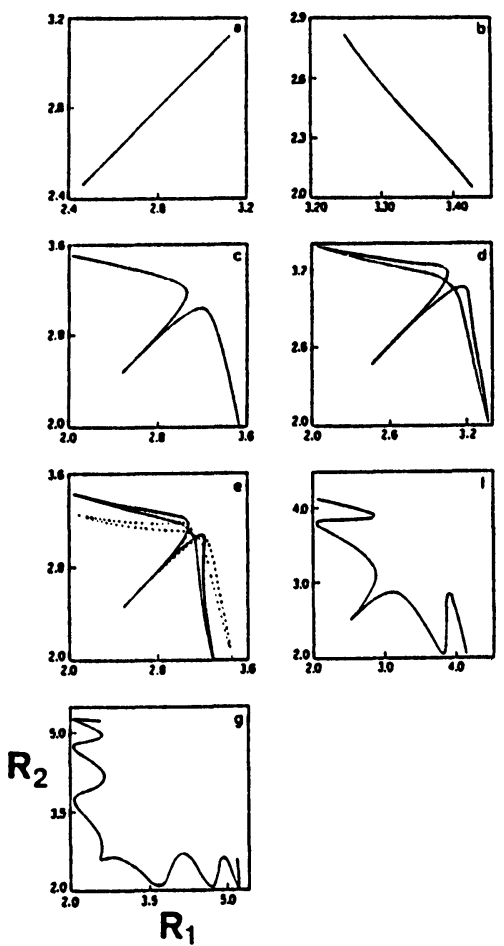

Figure 8 Periodic orbits located for the ozone potential. The periods are: (a) 47, (b) 26.5, (c) 150, (d) 108, (e) 216, (f) 230, and (g) 320 fs. Distances in Bohrs. 
(dash line) in time. Peaks labeled with (a) correspond to recurrence times which are multiples of the period of the symmetric stretch periodic orbit.

The symmetric stretch periodic orbit emerges from the saddle point of the potential which is a critical point of the Hamiltonian. ${ }^{33}$ It is unstable and remains unstable for an extended energy range. ${ }^{16}$ Therefore, no new periodic orbits bifurcate from it. For this reason, we consider the periodic orbits c,d,e,f, and g (Figure 8) to be of saddlenode type, although the high instability of the system does not allow a systematic investigation with the energy. ${ }^{32}$ The periodic orbit $b$ is asymmetric and it does not overlap with the initial wavepacket. ${ }^{16}$

In Figure 9 snapshots of the evolution of the wavepacket are shown. We observe that the first return in the initial region occurs after the reflection of the wavepacket from the potential along the symmetric stretch coordinate (Figure 9d), while the bulk of the wavepacket goes out to the exit channels. After that time more parts of the wavepacket are reflected back to the interaction region causing complex interferences. At later times most of the wavepacket has dissociated and what remains produces very complicated patterns (Figures $8 \mathrm{~h}-81$ ). In the calculations we have used an absorbing potential to remove the part of the wavepacket which reaches the edges of the grid.
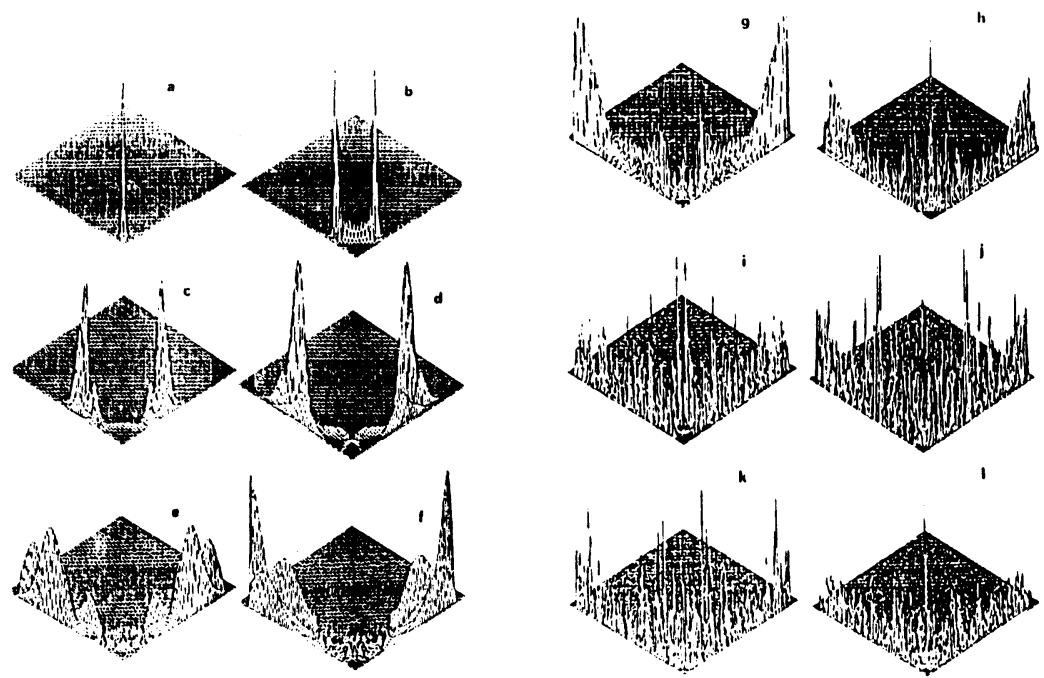

Figure 9 Snapshots of the time evolution of the wavepacket at (a) 0 , (b) 16.2 , (c) 32.5 , (d) 48.8 , (e) 65.1, (f) 81.3, (g) 110.3, (h) 165.5, (i) 220.6, (j) 275.8, (k) 331.0, and (l) $386.1 \mathrm{fs}$.

The recurrences in the correlation function are due to resonant states. In order to get an estimate of them we filter out the wavepacket at the times equal to the half or full periods of periodic orbits associated with the corresponding peaks in the correlation function. In other words, we use Equation 8 to evaluate the resonance states with $\hbar / \mathrm{E}_{n}=47,75$, and $108 \mathrm{fs}$ which correspond to the periods of periodic orbits a,c, and d, respectively. The results are shown in Figure 10. 

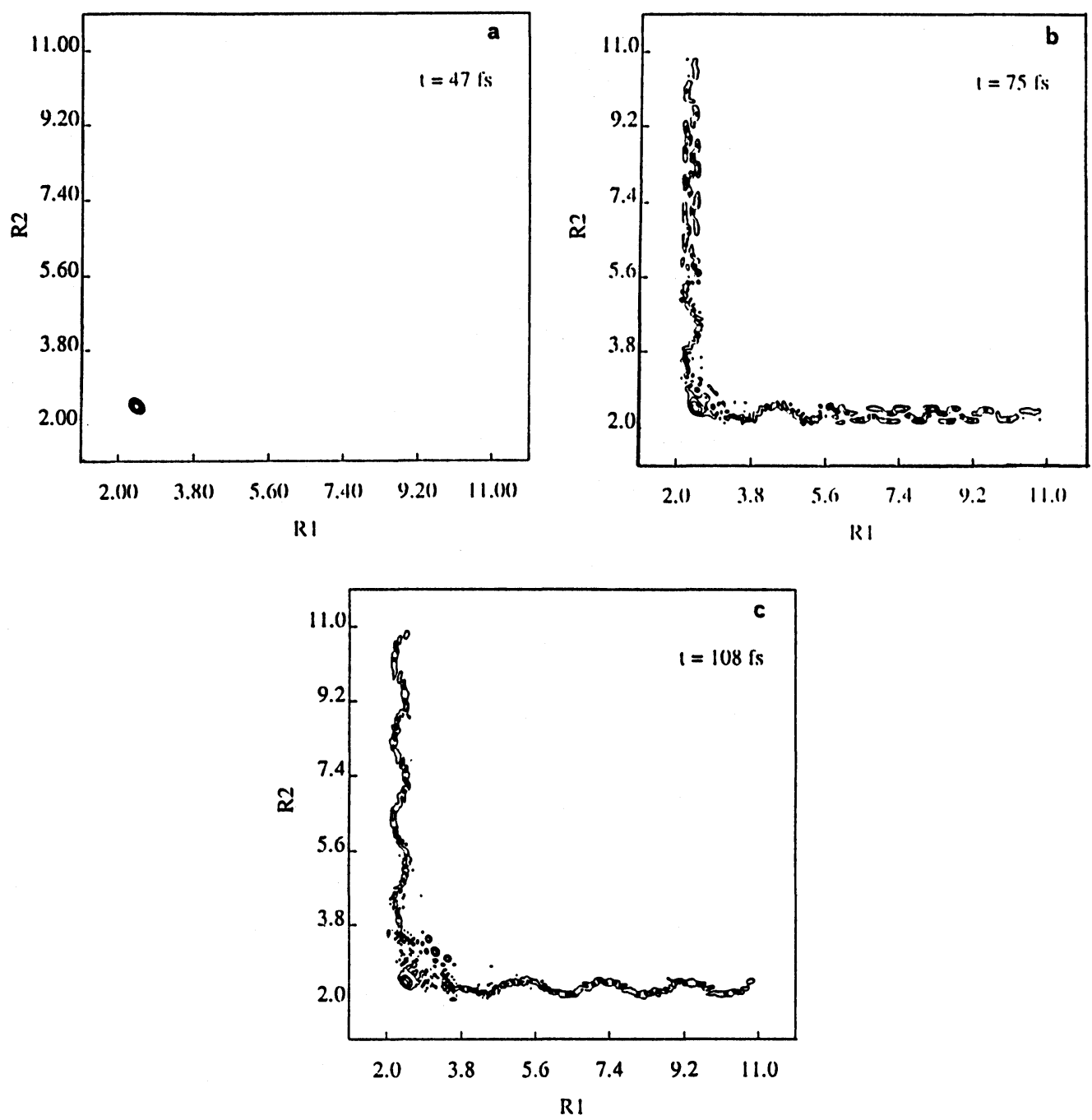

Figure 10 Resonance states associated with the three periodic orbits (a), (c), and (d) respectively.

We see that the probability density is concentrated in the initial interaction region, while a wave is propagated along the exit channels. Characteristically, in Figure 10c the highest probability density contours reproduce the pattern of the arrow and bow like periodic orbit.

We emphasize here that although the morphology of these resonances are extracted from the quantum mechanical calculations alone, however, they are predicted from the existence of the periodic orbits which emerge above the saddle point of the potential. 


\section{CONCLUSIONS}

By examining two specific examples, the excited electronic states of $\mathrm{SO}_{2}$ and of $\mathrm{O}_{3}$, we have shown that above the barriers of isomerization wavefunctions with a distinct morphology appear. This is mainly demonstrated for $\mathrm{SO}_{2}$ for which even the first vibrationally excited states show a deviation from the more common box like forms of two harmonic oscillators coupled with anharmonic terms.

The correspondence of the eigenfunctions to a particular type of periodic orbits provide an explanation for their topological characteristics. However, what we emphasize here is the role of the saddle-node type periodic orbits. These periodic orbits appear always in pairs with a stable and an unstable branch, and that is why they are important. Irrespectively to how irregular the phase space has been at lower energies, the emergence of the saddle-node periodic orbits will create a small region of stability (the case of ozone) or an extended area of stable motion (the case of $\mathrm{SO}_{2}$ ).

Is the saddle-node bifurcations a generic phenomenon for potentials with barriers of isomerizations? Although we have mathematical theorems which prove the existence of periodic orbits near critical points, the Weinstein-Moser theorems, ${ }^{37,38}$ we are not aware of a similar theorem for the saddle-node bifurcations. Studies in other systems, $\mathrm{ArCO},{ }^{29} \mathrm{HCN},{ }^{12} \mathrm{LiCN},{ }^{39} \mathrm{CO}_{2},{ }^{40,41}$ and $\mathrm{FH}_{2}{ }^{42}$ provide direct or indirect indications of the existence of saddle-node bifurcations and their role in assigning quantum eigenstates.

Characteristic examples are the van der Waals system $\mathrm{ArCO}^{29}$ and the repulsive potential surface of $\mathrm{CO}_{2}{ }^{40,41}$ In the latter, periodic orbits of similar type as those found for ozone were used to explain recurrences in the quantum autocorrelation function.

These results strengthen our view that saddle-node states are a generic phenomenon for molecules with potential barriers, and furthermore, prove the importance of periodic orbits in general, for understanding the complex dynamics of the molecular species at high vibrational energies.

\section{References}

1. See the articles in issue. J. Opt. Soc. Am., B 7, 1802 (1990).

2. M. Quack. Annu. Rev. Phys. Chem., 41, 839 (1990).

3. E. J. Heller. Acc. Chem. Res., 14, 368 (1981).

4. Z. Bacic and J. N. Light. In Ann. Rev. Phys. Chem., volume 40, page 469, 1989.

5. C. Leforestier, R. Bisseling, C. Cerjan, M. D. Feit, R. Friesner, A. Guldberg, A. Hammerich, G. Jolicard, W. Karrlein, H. D. Meyer, N. Lipkin, O. Roncero and R. Kosloff. J. Comput. Phys., 94, 59 (1991).

6. O. Bohigas, M.-J. Giannoni and C. Schmidt. Quantum Chaos and Statistical Nuclear Physics, Lecture Notes in Physics, volume 263, Springer-Verlag, 1986.

7. E. Abramson, R. W. Field, D. Imre, K. K. Innes and J. L. Kinsey. J. Chem. Phys., 83, 453 (1985).

8. H. Köppel, W. Domcke and L. S. Cederbaum. Advances in Chemical Physics, volume 57, John Wiley and Sons, Inc., 1984.

9. M. Broyer, G. Delacretaz, G.-Q. Ni, R. L. Whetten, J.-P. Wolf and L. Wöste. J. Chem. Phys., 90, 4620 (1989).

10. J. M. Gomez Llorente and H. S. Taylor.'J. Chem. Phys., 91, 953 (1989). 
11. X. Yang, C. A. Ragashi and A. M. Wodtke. J. Opt. Soc. Am., B 7, 1835 (1990).

12. S. C. Farantos and M. Founargiotakis. Chem. Phys., 142, 345 (1990).

13. S. C. Farantos, J. M. Gomez Llorente, O. Hahn and H. S. Taylor. J. Chem. Phys., 93, 76 (1990).

14. D. E. Freeman, K. Yoshino, J. R. Esmond and W. H. Parkinson. Planet. Space Sci., 32, 239 (1984).

15. B. R. Johnson and J. L. Kinsey. J. Chem. Phys., 91, 7638 (1989).

16. S. C. Farantos and H. S. Taylor. J. Chem. Phys., 94, 4887 (1991).

17. J. P. Pique, M. Joueux, J. Manners and G. Sitja. J. Chem. Phys., 95, 8744 (1991).

18. K. Yamanouchi, S. Takeuchi and S. Tsuchiya. J. Chem. Phys., 92, 4044 (1990).

19. M. C. Gutzwiller. Chaos in Classical and Quantum Mechanics, volume 1, Springer-Verlag, 1990.

20. E. Pollak and M. S. Child. Chem. Phys., 60, 23 (1981).

21. E. Pollak. Chem. Phys., 61, 305 (1981).

22. E. J. Heller. Phys. Rev. Letters, 53, 1515 (1984).

23. R. L. Waterland, J.-M. Yuan, C. C. Martens, R. E. Gillilan and W. P. Reinhardt. Phys. Rev. Letters 61, 2733 (1988).

24. H. Poincaré. Les Méthodes Nouevelles de la Méchanique Céleste, Gauthier-Villar, Paris, 1899.

25. R. Seydel. From Equilibrium to Chaos: Practical bifurcation and stability analysis, Elsevier, 1988.

26. E. Reithmeier. Periodic Solutions of Nonlinear Dynamical Systems, Lecture Notes in Mathematics, Springer-Verlag, 1991.

27. M. Founargiotakis, S. C. Farantos, G. Contopoulos and C. Polymilis. J. Chem. Phys., 91, 1389 (1989).

28. S. C. Farantos, M. Founargiotakis and C. Polymilis. Chem. Phys., 135, 347 (1989).

29. L. Zachilas and S. C. Farantos. Chem. Phys., 154, 55 (1991).

30. J.-M. Mao and J. B. Delos. Phys. Rev., A45, 1746 (1992).

31. G. Contopoulos. Astron. J., 75, 96 (1970).

32. S. C. Farantos. Chem. Phys., 159, 329 (1992).

33. S. C. Farantos. In Time Dependent Quantum Mechanics: Experiments and Theory, edited by J. Broeckhove, Plenum Co. Ltd, 1992.

34. M. D. Feit, J. A. Fleck and A. Steiger. J. Comput. Phys., 47, 412 (1982).

35. A. R. Hoy and J. C. D. Brand. Mol. Phys., 36, 1409 (1978).

36. F. L. Quéré and C. Leforestier. J. Chem. Phys., 94, 1118 (1991).

37. A. Weinstein. Inv. Math., 20, 47 (1973).

38. J. Moser. Commun. Pure Appl. Math., 29, 727 (1976).

39. J. M. Gomez Llorente, F. Borondo, N. Berenguer and R. M. Benito. Chem. Phys. Letters, 192, 430 (1992).

40. K. C. Kulander, C. Cerjan and A. E. Orel. J. Chem. Phys., 94, 2571 (1991).

41. R. Schinke and V. Engel. J. Chem. Phys., 93, 3252 (1990).

42. O. Hahn and H. S. Taylor. J. Chem. Phys., 96, 5915 (1992). 